\title{
Derivation and characterization of new Chinese human embryonic stem cell lines with normal and abnormal karyotypes
}

Xiaofang Sun ${ }^{1}$, Yifei Yin ${ }^{1}$, Yonghua Jiang ${ }^{1}$, Xiaolin Long ${ }^{1}$, Hongzi Du ${ }^{1}$, Wenhong Zhang ${ }^{1}$, Xinjie Chen ${ }^{1}$, Weihua Wang ${ }^{1}$, Guohong Xiao ${ }^{1}$

${ }^{1}$ Institute of Gynecology and Obstetrics, The Third Affiliated Hospital of Guangzhou Medical College, Duobao Road, Guangzhou, China

This study was designed to investigate an effective method for the derivation of human embryonic stem cells (hESC) from poor quality embryos discarded from in vitro fertilization (IVF) laboratories. Poor quality embryos were donated from IVF center on day 3 and cultured in a blastocyst medium for 2 days, and then in a blastocyst optimum culture medium for additional 2 days. The isolated inner cell masses (ICM) were inoculated onto the feeder layer of mouse embryonic fibroblasts for subcultivation. The biological characterizations and differentiation capability of these cells were examined after at least 15 passages. Forty-two embryos developed to expanded blastocysts or hatched blastocysts and ICMs were successfully isolated from all embryos. Six new hESC lines were established after further culture. All hESC lines were positive for stage-specific embryonic antigen SSEA-4, TRA-1-81, TRA-1-60 markers and negative for SSEA-1 marker. They showed a high level of alkaline phosphatase activity, positive expression of transcription factor Oct-4. Furthermore, these cells had the potential to form both embryoid bodies in vitro and teratomas in vivo. Five hESC lines had normal karyotypes and one had trisomy 13. In this paper we described a stable and effective system for hESC isolation and established six new hESC lines, which will provide an excellent experimental model for cell therapy, developmental biology and genetic research.

Keywords: embryonic stem cells, human blastocyst, inner cell mass, mechanical isolation, immunosurgery

Cell Research (2008) 18:s33. doi: 10.1038/cr.2008.123; published online 4 August 2008

Correspondence: Xiaofang Sun

E-mail:xiaofangsun@hotmail.com

Xiaofang Sun is the current director of Guangzhou Municiple Key laboratory of Reproduction and Genetics. She is current Associate Professor in Guangzhou Medical College. Sun is a key member of Medical Genetics, Guangdong Branch of CMA and a member of Guangdong Committee of CPPCC. She is recognized as an outstanding scientist in Guangzhou and was honored as the outstanding expert which enjoys the State Council special allowance since 2000. She obtained the Guangzhou health enterprise tenth five-year plan key research project entitled "studies on embryonic stem cells" from the national natural sciences foundation and the Guangdong Province tenth five-year plans. In recent years she was awarded the science and technology progress third prize by Guangdong Province twice, the Guangzhou science and technology progress second prize twice, third prize twice. Prof. Sun's research focuses on embryonic stem (ES) cells, Genetics, Immunology and Assist Reproduction Technique (ART), and involved in the success of the first two babies in in-vitro fertilization (IVF) program of Guangdong province. 